\title{
On Throughput Improvement using Immediate Re-transmission in Grant-Free Random Access with Massive MIMO
}

\author{
Jinho Choi
}

\begin{abstract}
To support machine-type communication (MTC), massive multiple-input multiple-output (MIMO) has been considered for grant-free random access. In general, the performance of grant-free random access with massive MIMO is limited by the number of preambles and the number of active devices. In particular, when there are a number of active devices transmitting data packets simultaneously, the signal-to-interference-plus-noise ratio (SINR) cannot be high enough for successful decoding. In this paper, in order to improve performance, we consider immediate re-transmissions for an active device that has a low SINR although it does not experience preamble collision to exploit re-transmission diversity (RTD) gain. To see the performance of the proposed approach, we perform throughput analysis with certain approximations and assumption. Since the proposed approach can be unstable due to immediate re-transmissions, conditions for stable systems are also studied. Simulations are carried out and it is shown that analysis results reasonably match simulation results.
\end{abstract}

Index Terms-Grant-free Random Access; Massive MIMO Retransmission; Throughput Analysis

\section{INTRODUCTION}

In order to provide devices' connectivity for the Internet of Things (IoT), a number of different approaches are considered [1]. Among those, for wide area coverage, machine-type communication (MTC) in 5th generation (5G) cellular systems has been actively studied [2] [3]. In MTC, although there are a large number of devices to be connected, in general, they have short data packets with sporadic activity. Thus, random access based on ALOHA [4] is employed for MTC thanks to low signaling overhead [5] [6] [7] [8].

To support massive connectivity for a large number of devices and sensors within a cellular system (e.g., 5G), massive multiple-input multiple-output (MIMO) [9] can be considered with a base station (BS) equipped with a large number of antenna elements. According to [10], massive MIMO would be a promising solution to massive MTC, as the capacity becomes unbounded in the presence of pilot contamination, which can support a very large number of devices in each cell. In massive MIMO, the channel state information (CSI) of each device is characterized by a random channel vector. Thus, as long as the channel vectors of active devices, which are the devices with data packets to transmit, are different (and nearly orthogonal with a sufficiently large number of antennas), the

The author is with the School of Information Technology, Deakin University, Geelong, VIC 3220, Australia (e-mail: jinho.choi@deakin.edu.au). This research was supported by the Australian Government through the Australian Research Council's Discovery Projects funding scheme (DP200100391).
BS is able to detect/decode simultaneously transmitted signals from them. Thus, for random access with massive MIMO, it is crucial to allow the BS to estimate the active devices' channel vectors. To this end, an active device needs to transmit a preamble prior to transmission of data packet. Furthermore, no handshaking protocol is required to allocate channel resources for transmissions of data packets from active devices thanks to high spatial resolution or selectivity in massive MIMO. As a result, various grant-free random access schemes with massive MIMO are studied in [11] [12] [13] [14] [15].

In most grant-free random access schemes with massive MIMO have two phases. In the first phase, each active device sends a preamble that is randomly chosen from a predetermined preamble pool (i.e., a finite set of sequences) so that the BS is able to estimate active devices' channel vectors. In the second phase, the active devices transmit their data packets and the BS performs beamforming with estimated channel vectors to decode data packets from multiple active devices.

In grant-free random access with massive MIMO, as mentioned earlier, each active device transmits a preamble. Thus, the BS needs to detect transmitted preambles, which is called device activity detection [12] [13]. In addition, as discussed in [16] [17] [18], preamble design plays a key role in providing a good performance in terms of the probability of successful decoding or success probability.

Provided that the BS is able to detect transmitted preambles without errors and a set of orthogonal preambles are used, the performance of grant-free random access with massive MIMO is mainly dependent on the number of preambles and the number of active devices. Since the number of preambles is finite, there is a non-zero probability that multiple active devices choose the same preamble, which leads to preamble collision (PC). Since the estimated channel vector is a noisy superposition of multiple channels vectors of the active devices that choose the same preamble, the signal-to-interferenceplus-noise ratio (SINR) after beamforming becomes poor and subsequent decoding for data packet is likely unsuccessful.

As discussed in [15], with massive MIMO, the error probability of preamble detection is negligible. Thus, the BS can broadcast the outcomes of preamble detection so that active devices with PC do not transmit their data packets, which can result in a high SINR of the other active devices without PC and an improved throughput. However, if the number of the active devices without PC is large, the resulting SINR can be low. Thus, the performance is also limited by the number of active devices (even if the number of preambles is sufficiently 
large for a low probability of PC).

In this paper, we propose a grant-free random access scheme with massive MIMO, where an active device that does not experience PC, but has a low SINR performs immediate re-transmissions. Since the active device's channel vector is successfully estimated by the BS (as PC does not happen), this device can re-transmit data packets without sending additional preambles. This approach is advantageous for forthcoming active devices, since the probability of the PC in subsequent slots is not increased by this active device. In addition, since multiple copies of a data packet are received by immediate re-transmissions, the BS can have re-transmission diversity (RTD) gain that can increase the SINR. As a result, after a certain number of immediate re-transmissions, the BS can eventually decode the data packet.

Due to immediate re-transmissions, the proposed system can be unstable if the number of re-transmissions is unbounded. Thus, we study the stability issue of the proposed system with the throughput analysis. Unfortunately, there are a number of difficulties. In particular, since the SINR is a random variable that depends on the number of active devices (although the channel hardening effect of massive MIMO is taken into account [9] [19]) and increased by RTD (via immediate retransmissions), the throughput analysis is not straightforward. Thus, for tractable analysis, we consider approximations of SINR with immediate re-transmissions and study FosterLyapunov criteria [20] [21], which allow us to derive key conditions for stable systems. Simulations are carried out and it is shown that the analysis results reasonably agree with simulation results.

In summary, the key contributions of the paper are i) a grant-free random access scheme with massive MIMO is proposed using immediate re-transmissions to improve the throughput; ii) its throughput analysis is carried out using Foster-Lyapunov criteria to find key conditions for stable systems and throughput under certain conditions.

The rest of the paper is organized as follows. In Section II, we present the system model for grant-free random access with massive MIMO. In Section III, a simple expression for the SINR with estimated channel vector is derived to be used for throughput analysis. We discuss different types of feedback signals in grant-free random access with massive MIMO and propose a modified approach that can improve the throughput using immediate re-transmissions in Section IV. In Section V, the throughput is analyzed using certain approximations and assumptions and the stability issue of the proposed approach is addressed. In Section VI, we present simulation results that are also compared with the analysis results obtained in Section V. The paper is concluded in Section VII with some remarks.

Notation: Matrices and vectors are denoted by upper- and lower-case boldface letters, respectively. The superscripts $\mathrm{T}$ and $\mathrm{H}$ denote the transpose and complex conjugate, respectively. $\lfloor x\rfloor$ represents the greatest integer that is less than or equal to $x$, and $\lceil x\rceil$ denote the least integer that is greater than or equal to $x . \mathbb{E}[\cdot]$ and $\operatorname{Var}(\cdot)$ denote the statistical expectation and variance, respectively. $\mathcal{C N}(\mathbf{a}, \mathbf{R})$ represents the distribution of circularly symmetric complex Gaussian (CSCG) random vectors with mean vector a and covariance matrix $\mathbf{R}$.

\section{SySTEM MODEL}

Suppose that a grant-free random access system consists of a BS and multiple devices. It is assumed that the BS is equipped with $M$ antenna elements and each device has a single antenna.

For grant-free random access, we consider two phases [14] [16]. The first phase is the preamble transmission phase and the following one is the data transmission phase. Throughout the paper, it is assumed that a slot is divided into two subslots, where preambles are transmitted in the first sub-slot and data packets are transmitted in the second sub-slot. In the preamble transmission phase (during the first sub-slot), each active device with data is to send a randomly selected preamble from the following preamble pool:

$$
\mathcal{C}=\left\{\mathbf{c}_{1}, \ldots, \mathbf{c}_{L}\right\}
$$

where $\mathbf{c}_{l} \in \mathbb{C}^{N \times 1}$ represents the $l$ th preamble. Throughout the paper, it is assumed that the $\mathbf{c}_{l}$ 's are orthonormal sequences of length $N$. Thus, we assume that $L=N$. After sending a preamble, an active device sends its data packet in the data transmission phase. Throughout the paper, it is assumed that the lengths of data packets of all devices are the same. In addition, all the devices are synchronized (to this end, the BS needs periodically broadcast a beacon signal).

Let $\mathbf{h}_{k} \in \mathbb{C}^{M \times 1}$ represent the channel vector from active device $k$ to the BS. Then, in the preamble transmission phase, the $\mathrm{BS}$ receives the following signal in the space and time domain:

$$
\mathbf{Y}=\sum_{k=1}^{K} \mathbf{h}_{k} \sqrt{P_{k}} \mathbf{c}_{l(k)}^{\mathrm{T}}+\mathbf{N} \in \mathbb{C}^{M \times N},
$$

where $K$ represents the number of active devices, $l(k)$ denotes the index of the preamble chosen by active device $k, P_{k}$ is the transmit power of active device $k$, and $[\mathbf{N}]_{m, n} \sim \mathcal{C N}\left(0, N_{0}\right)$ is the background noise at the $m$ th antenna and the $n$th preamble symbol duration.

Each active device transmits its data packet of length $D$ during the data transmission phase (in the second sub-slot). The corresponding received signal at the BS becomes

$$
\mathbf{Z}=\sum_{k=1}^{K} \mathbf{h}_{k} \sqrt{P_{k}} \mathbf{b}_{k}^{\mathrm{T}}+\tilde{\mathbf{N}} \in \mathbb{C}^{M \times D},
$$

where $\mathbf{b}_{k}$ represents the data packet from active device $k$ and $[\tilde{\mathbf{N}}]_{m, d} \sim \mathcal{C N}\left(0, N_{0}\right)$ is the background noise at the $m$ th antenna and the $n$th data symbol duration. Throughout the paper, we assume that $\mathbb{E}\left[\mathbf{b}_{k}\right]=\mathbf{0}$ and $\mathbb{E}\left[\mathbf{b}_{k} \mathbf{b}_{k}^{\mathrm{H}}\right]=\mathbf{I}$ (i.e., the symbol energy is normalized).

The BS uses the correlator to estimate the channel vector as follows:

$$
\begin{aligned}
\mathbf{g}_{l} & =\mathbf{Y} \mathbf{c}_{l}=\sum_{k=1}^{K} \mathbf{h}_{k} \sqrt{P_{k}} \delta_{l(k), l}+\mathbf{N} \mathbf{c}_{l} \\
& =\sum_{k \in \mathcal{K}_{l}} \mathbf{h}_{k} \sqrt{P_{k}}+\mathbf{N} \mathbf{c}_{l},
\end{aligned}
$$


where $\delta_{l, l^{\prime}}$ is the Kronecker delta (i.e., $\delta_{l, l^{\prime}}=1$ if $l=l^{\prime}$, and 0 otherwise) and $\mathcal{K}_{l}$ represents the index set of the active devices that choose preamble $l$. If active device $k$ is the only device that chooses preamble $l$ (i.e., $l(k)=l$ or $\mathcal{K}_{l}=\{k\}$ ), thanks to the orthogonality of preambles, it can be shown that

$$
\mathbf{g}_{l}=\mathbf{h}_{k} \sqrt{P_{k}}+\mathbf{n}_{l},
$$

where $\mathbf{n}_{l}=\mathbf{N} \mathbf{c}_{l} \sim \mathcal{C N}\left(\mathbf{0}, N_{0} \mathbf{I}\right)$. To decode the data packet from active device $k$, conjugate beamforming is applied and the output of the beamformer becomes

$$
\begin{aligned}
\mathbf{x}_{l} & =\mathbf{g}_{l}^{\mathrm{H}} \mathbf{Z} \\
& =\mathbf{g}_{l}^{\mathrm{H}} \mathbf{h}_{k} \sqrt{P_{k}} \mathbf{b}_{k}^{\mathrm{T}}+\sum_{k^{\prime} \neq k} \mathbf{g}_{l}^{\mathrm{H}} \mathbf{h}_{k^{\prime}} \sqrt{P_{k^{\prime}}} \mathbf{b}_{k^{\prime}}^{\mathrm{T}}+\mathbf{g}_{l}^{\mathrm{H}} \tilde{\mathbf{N}} .
\end{aligned}
$$

If $\left|\mathbf{g}_{l}^{\mathrm{H}} \mathbf{h}_{k}\right|^{2}$ is sufficiently larger than $\left|\mathbf{g}_{l}^{\mathrm{H}} \mathbf{h}_{k^{\prime}}\right|^{2}$, a high SINR can be achieved for successful decoding.

\section{SINR With Estimated CHANNEL VECTORS}

In this section, we consider a simple expression for the SINR in the grant-free random access system in Section II, which allows tractable analysis in Section V. [22].

For tractable analysis, we consider the following assumption

A) Throughout the paper, $P_{k}$ is decided to be inversely proportional to $\ell_{k}$ via power control so that

$$
\mathbf{h}_{k} \sqrt{P_{k}}=\mathbf{v}_{k} \sqrt{P_{\mathrm{rx}}}
$$

where $P_{\mathrm{rx}}$ represents the (average) receive signal power and $\mathbf{v}_{k} \sim \mathcal{C N}(\mathbf{0}, \mathbf{I})$ is independent for all $k$ (i.e., Rayleigh fading is assumed for small-scale fading).

As in [23] [24], when no power control is employed, each device may have a different receive signal power at the BS that can be exploited for collision resolution. However, as mentioned above, we do not consider this case in this paper, and $\mathrm{PC}$ is declared if there are multiple active devices that choose the same preamble, which might lead to a worse performance than the case where different receive signal powers (due to no power control) are exploited for collision resolution.

For convenience, let $\gamma=\frac{P_{\mathrm{rx}}}{N_{0}}$ be the signal-to-noise ratio (SNR). For given $\left\{\mathbf{v}_{k}\right\}$, to find the SINR, we consider active device $k$ with $\mathcal{K}_{l}=\{k\}$ (i.e., without PC). From (6), it can be shown that

$$
\begin{aligned}
\mathbf{x}_{l}= & \left(P_{\mathrm{rx}}\left\|\mathbf{v}_{k}\right\|^{2}+\sqrt{P_{\mathrm{rx}}} \mathbf{n}_{l}^{\mathrm{H}} \mathbf{v}_{k}\right) \mathbf{b}_{k}^{\mathrm{T}} \\
& +\sum_{k^{\prime} \neq k}\left(P_{\mathrm{rx}} \mathbf{v}_{k}^{\mathrm{H}} \mathbf{v}_{k^{\prime}}+\sqrt{P_{\mathrm{rx}}} \mathbf{n}_{l}^{\mathrm{H}} \mathbf{v}_{k^{\prime}}\right) \mathbf{b}_{k^{\prime}}^{\mathrm{T}} \\
& +\left(\sqrt{P_{\mathrm{rx}}} \mathbf{v}_{k}+\mathbf{n}_{l}\right)^{\mathrm{H}} \tilde{\mathbf{N}} .
\end{aligned}
$$

For given $\left\{\mathbf{v}_{k}\right\}$, the conditional SINR of active device $k$ without PC becomes

$$
\operatorname{SINR}_{k}=\frac{\gamma^{2}\left\|\mathbf{v}_{k}\right\|^{2}+\gamma}{I_{k}}
$$

where

$$
I_{k}=\sum_{k^{\prime} \neq k}\left(\gamma^{2} \frac{\left|\mathbf{v}_{k}^{\mathrm{H}} \mathbf{v}_{k^{\prime}}\right|^{2}}{\left\|\mathbf{v}_{k}\right\|^{2}}+\gamma \frac{\left\|\mathbf{v}_{k^{\prime}}\right\|^{2}}{\left\|\mathbf{v}_{k}\right\|^{2}}\right)+\gamma+\frac{M}{\left\|\mathbf{v}_{k}\right\|^{2}} .
$$

In (9), the signal power and interference-plus-noise power terms are obtained by taking the expectation with respect to the noise terms, and the term $\left(\sqrt{P_{\mathrm{rx}}} \mathbf{n}_{k}^{\mathrm{H}} \mathbf{v}_{k}\right) \mathbf{b}_{k}^{\mathrm{T}}$ in (8) is part of the desired signal (i.e., $\mathbf{g}_{l}^{\mathrm{H}} \mathbf{h}_{k} \sqrt{P_{k}} \mathbf{b}_{k}^{\mathrm{T}}$ in (6)). As a result, its power term, i.e., $\gamma$, becomes the 2 nd term in the numerator in (9).

Based on the strong law of large numbers, since $\frac{\left\|\mathbf{v}_{k}^{\mathrm{H}}\right\|^{2}}{M} \rightarrow 1$ (w.p. 1) as $M \rightarrow \infty$, it can be shown that

$$
I_{k} \rightarrow \sum_{k^{\prime} \neq k} \gamma^{2}\left|X_{k, k^{\prime}}\right|^{2}+(K-1) \gamma+\gamma+1,
$$

where $X_{k, k^{\prime}}=\frac{\mathbf{v}_{k}^{\mathrm{H}} \mathbf{v}_{k^{\prime}}}{\left\|\mathbf{v}_{k}\right\|}$. For given $\mathbf{v}_{k}, X_{k, k^{\prime}}$ is seen as a weighted sum of independent CSCG random variables. Thus, for given $\mathbf{v}_{k}, X_{k, k^{\prime}} \sim \mathcal{C N}\left(0, \sum_{m=1}^{M} \frac{\left|\left[\mathbf{v}_{k}\right]_{m}\right|^{2}}{\left\|\mathbf{v}_{k}\right\|^{2}}\right)$. Since $\sum_{m=1}^{M} \frac{\left|\left[\mathbf{v}_{k}\right]_{m}\right|^{2}}{\| \mathbf{v}_{k}||^{2}}=1$, we can see that $X_{k, k^{\prime}} \sim \mathcal{C} \mathcal{N}(0,1)$, which is independent of $\mathbf{v}_{k}$. Furthermore, for given $\mathbf{v}_{k}, X_{k, k^{\prime}}$, $k^{\prime} \neq k$, are independent. Then, it can be shown that

$$
\lim _{M \rightarrow \infty} \mathbb{E}\left[I_{k}\right]=\gamma^{2}(K-1)+\gamma K+1,
$$

while

$$
\lim _{M \rightarrow \infty} \frac{\gamma^{2}\left\|\mathbf{v}_{k}\right\|^{2}+\gamma}{M}=\gamma^{2}
$$

Thus, it can be shown that

$$
\lim _{M \rightarrow \infty} \frac{\mathbb{E}\left[\text { SINR }_{k}\right]}{M}=\frac{1}{(K-1)+\frac{K+\frac{1}{\gamma}}{\gamma}} .
$$

With a sufficiently large $M$ (but finite), for a given $K$, the conditional mean of SINR (with estimated channel vectors) can be approximated as follows:

$$
\operatorname{SINR}_{k}(K)=\frac{M}{K\left(1+\frac{1}{\gamma}\right)+\frac{1}{\gamma^{2}}-1}=\frac{M}{K b_{1}+b_{0}},
$$

which shows that the SINR decreases with $K$. Here, $b_{1}=1+\frac{1}{\gamma}$ and $b_{0}=\frac{1}{\gamma^{2}}-1$, which are independent of $M$ and $K$. Note that while the SINR expression in (15) or similar ones can be found in the literature (e.g., [25] [14]), it has been (re-)derived to be used in Section $\mathrm{V}$ for the throughput and stability of the proposed approach.

In Fig. 1, empirical results of SINR are shown with the average SINR in (15) for different values of $K$ when $M=100$ and $\gamma=10 \mathrm{~dB}$. It is noteworthy that the SINR in (15) is dependent on $K$ that can be seen as a random variable in random access.

\section{Feedback Signals and Immediate RE-TRANSMISSIONS}

In grant-free random access with massive MIMO, an active device can successfully transmit a packet if $i$ ) there is no $\mathrm{PC}$ and ii) the SINR is sufficiently high, i.e., $\mathrm{SINR}_{k} \geq \Gamma$, where $\Gamma$ is a threshold SINR for successful decoding. As a result, although $\mathrm{PC}$ does not happen, an active device needs to drop the packet or re-transmit (after a random delay) when the SINR is lower than $\Gamma$ (as decoding is unsuccessful), which can degrade throughput. In order to improve throughput, re-transmission diversity (RTD) [26] can be employed with 


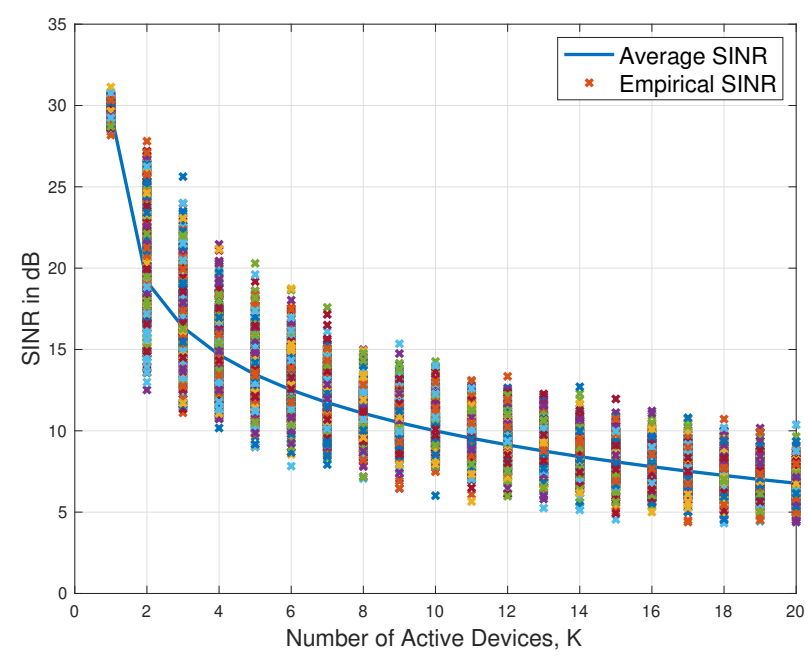

Fig. 1. Average SINR with empirical results (with the estimated channel vectors) as functions of $K$ when $M=100$ and $\gamma=10 \mathrm{~dB}$.

two different types of feedback signals. In this section, we discuss feedback signals and propose an approach for grantfree random access with massive MIMO based on RTD.

\section{A. Feedback after Preamble Transmission}

If there are $K$ active devices that randomly choose the preambles in $\mathcal{C}$, the probability of $\mathrm{PC}$, which is the conditional probability that an active device experiences $\mathrm{PC}$ provided that there are $K$ active devices, is given by

$$
\mathbb{P}_{\mathrm{pc}}(K)=1-\left(1-\frac{1}{L}\right)^{K-1}=1-\alpha_{L}^{K-1},
$$

where $\alpha_{L}=1-\frac{1}{L}$. In general, the channel vectors of the active devices with PC cannot be correctly estimated. To see this, suppose that the $l$ th preamble is chosen by active devices 1 and 2. Then, from (4), the output of the correlator becomes

$$
\mathbf{g}_{l}=\mathbf{v}_{1}+\mathbf{v}_{2}+\mathbf{n}_{l} .
$$

Thus, we can assume that the active devices with PC have low SINRs, while the signals from them become interfering signals that degrade the SINR of the active devices without PC as shown in (15). For convenience, the active devices without $\mathrm{PC}$ are referred to as PC-free (active) devices.

In order to improve the performance, the BS can detect PCs and send a feedback signal after the preamble transmission phase to inform the collided preambles. That is, for each preamble, acknowledgment (ACK) or negative acknowledgment (NACK) is sent. The resulting feedback signal is referred to as feedback signal of Type I. With feedback signal of Type I, the active devices with PC do not transmit their packets (their packets are backlogged for re-transmissions according to re-transmission policies or dropped). Thus, for given $K$, the average number of PC-free devices is given by

$$
\bar{K}(K)=K \alpha_{L}^{K-1} .
$$

In (15), $K$ can be replaced with $\bar{K}(K)$, which will be simply denoted by $\bar{K}$, for the SINR if the active devices with PC do not transmit their data packets, which leads to a higher SINR (because $\bar{K} \leq K$ ) [15].

\section{B. Feedback after Data Packet Transmission and RTD}

For a PC-free device, decoding of data packet can fail if its SINR is not sufficiently high. For successful decoding, as mentioned earlier, the SINR has to be higher than or equal to a threshold, $\Gamma$. When the SINR is not sufficiently high or decoding is not successful, the data packet of a PC-free device can be dropped or backlogged for re-transmission after a random delay. For convenience, the resulting random access system is referred to as the conventional system.

For a PC-free device, although its data packet cannot be decoded, the BS knows its channel vector. Thus, we can take advantage of known channel vector and exploit RTD by allowing a PC-free device to continuously re-transmit the same packet until decoding becomes successful to improve throughput as shown in Fig. 2, where active device 1 performs $(Q+1)$-time transmissions of a data packet. Clearly, since its channel is estimated by the preamble transmission in the first slot, the PC-free device does not need to send any preambles in the subsequent slots (this helps reduce the PC for next active devices, e.g., active device 2 in Fig. 2). For convenience, the packet of a PC-free device that is to be re-transmitted in subsequent slots is referred to as a packet in service (PiS). Furthermore, the packet in the $q$ th subsequent slot for retransmission is referred to as the $q$ th PiS.

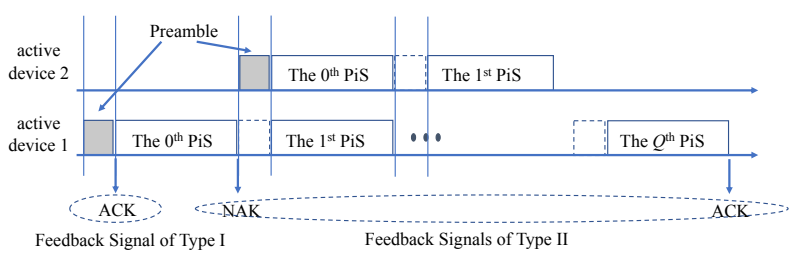

Fig. 2. An illustration of packet transmissions in grant-free random access with IRT where two active devices transmit their packets with preambles.

There are few remarks as follows.

- To exploit the known channels of PC-free devices, all the re-transmissions have to be done within a coherence time. Thus, in practice, the maximum ${ }^{1}$ number of retransmissions has to be limited, although it is not considered in this paper. Furthermore, the BS can fail to decode after receiving the maximum number of re-transmissions (e.g., due to a low accumulated SINR). In this case, the BS can send NACK of Type I to the device so that it can try to send the data packet later (according to a retransmission policy) or drops.

- Due to the re-transmissions of data packet, the SINR can increase and after a certain number of re-transmissions, the BS can succeed to decode the data packets. For retransmissions, the BS needs to send ACK or NACK feedback signal (for success or failure of decoding), which is referred to as Feedback Signal of Type II in this

\footnotetext{
${ }^{1}$ In this paper, we assume that the mobility of devices in MTC is limited and the coherence time is sufficiently long for a number of re-transmissions.
} 
paper. In addition, the resulting random access system is referred to as the immediate re-transmission (IRT) based system or IRT system.

- Since a PC-free device in the IRT system can transmit more than one data packet (i.e., multiple PiSs), the SINR can be low and a lower SINR results in more re-transmissions. As a result, the IRT system can be unstable (i.e., the number of re-transmissions becomes unbounded). In the next section, we address the stability issue of IRT through throughput analysis.

\section{Throughrut Analysis}

In this section, we study the throughput of the IRT system. For tractable analysis, we assume that the arrivals at each slot are independent and follow a Poisson distribution with mean arrival rate $\lambda$ (in the number of new active devices per slot), i.e.,

$$
K \sim \operatorname{Pois}(\lambda)
$$

Throughout this section, we assume that an active device drops its data packet when it receives NACK of Type I.

\section{A. Conventional System}

In the conventional system, for given $K$ in a slot, the average number of dropped packets due to PC as well as low SINR is $\mathbb{E}[U(K) \mid K]$, where

$$
U(K)=K\left(1-\alpha_{L}^{K-1}\right)+\bar{K} \mathbb{1}\left(\operatorname{SINR}_{k}<\Gamma\right) .
$$

In (19), in order to obtain the mean of the second term on the right-hand side (RHS), the distribution of the SINR is required. Although it might be possible to find the distribution of the SINR, for tractable analysis, we use the conditional mean of SINR in (15), which is a function of $\bar{K}$, in this subsection.

Assume that $\bar{K}$ is a Poisson random variable with the following mean:

$$
\bar{\lambda}=\mathbb{E}[\bar{K}]=\sum_{k=0}^{\infty} k \alpha_{L}^{k-1} p_{\lambda}(k)=\lambda e^{-\frac{\lambda}{L}},
$$

where $p_{\lambda}(k)=\frac{e^{-\lambda} \lambda^{k}}{k !}$. Then, it can be shown that

$$
\begin{aligned}
\mathbb{E}\left[\bar{K} \mathbb{1}\left(\operatorname{SINR}_{k}(\bar{K})<\Gamma\right)\right] & =\sum_{k=0}^{\infty} k \mathbb{1}\left(\frac{M}{k b_{1}+b_{0}}<\Gamma\right) p_{\bar{\lambda}}(k) \\
& =\sum_{k>K_{\Gamma}} k p_{\bar{\lambda}}(k) \\
& =\bar{\lambda}-\sum_{\left.K_{\Gamma}\right\rfloor}^{k} k p_{\bar{\lambda}}(k) \\
& =\bar{\lambda}-\bar{F}_{\bar{\lambda}}\left(\left\lfloor K_{\Gamma}\right\rfloor-1\right),
\end{aligned}
$$

where $F_{\bar{\lambda}}(n)=\sum_{k=0}^{n} \frac{e^{-\bar{\lambda}} \bar{\lambda}^{k}}{k !}$ is the cumulative distribution function (cdf) with mean $\bar{\lambda}$ and

$$
K_{\Gamma}=\frac{1}{b_{1}}\left(\frac{M}{\Gamma}-b_{0}\right) .
$$

Clearly, $K_{\Gamma}$ is a constant that is independent of $K$. From (19) and (21), it can be shown that

$$
\begin{aligned}
\lambda_{\mathrm{d}} & \triangleq \mathbb{E}[U(K)]=\mathbb{E}[\mathbb{E}[U(K) \mid K]] \\
& =\lambda-\bar{\lambda}+\bar{\lambda}-\bar{\lambda} e^{-\bar{\lambda} \sum_{k=0}^{\left\lfloor K_{\Gamma}\right\rfloor-1} \frac{\bar{\lambda}^{k}}{k !}} \\
& =\lambda-\bar{\lambda} e^{-\bar{\lambda}} \sum_{k=0}^{\left\lfloor K_{\Gamma}\right\rfloor-1} \frac{\bar{\lambda}^{k}}{k !} .
\end{aligned}
$$

Let

$$
\lambda_{\mathrm{n}}=\lambda-\lambda_{\mathrm{d}}=\bar{\lambda} F_{\bar{\lambda}}\left(\left\lfloor K_{\Gamma}\right\rfloor-1\right),
$$

where $\lambda_{\mathrm{n}}$ represents the average number of successfully transmitted packets per slot or throughput. Thus, the maximum throughput of the conventional system becomes

$$
\begin{aligned}
\eta_{\text {con }} & =\max _{\lambda} \bar{\lambda} F_{\bar{\lambda}}\left(\left\lfloor K_{\Gamma}\right\rfloor-1\right) \\
& =\max _{0 \leq \bar{\lambda} \leq L e^{-1}} \bar{\lambda} F_{\bar{\lambda}}\left(\left\lfloor K_{\Gamma}\right\rfloor-1\right),
\end{aligned}
$$

because $\bar{\lambda} \leq \max _{\lambda} \lambda e^{-\frac{\lambda}{L}}=L e^{-1}$. In addition, let

$$
\lambda_{\text {con }}=\underset{\lambda}{\operatorname{argmax}} \bar{\lambda} F_{\bar{\lambda}}\left(\left\lfloor K_{\Gamma}\right\rfloor-1\right) \text {. }
$$

The maximum throughput of the conventional system can approach $L e^{-1}$ if $K_{\Gamma}$ is sufficiently large. Clearly, for a fixed $\Gamma$, as $M \rightarrow \infty, K_{\Gamma} \rightarrow \infty$, which implies that $\eta_{\text {con }} \rightarrow L e^{-1}$, i.e., the performance of the conventional system with a sufficiently large $M$, is limited by the number of preambles, $L$. However, in practice, since $M$ is finite, it is expected that $\eta_{\text {con }}$ is lower than $L e^{-1}$.

\section{B. Immediate Re-Transmission based System}

In the IRT system, only PC results in dropped packets. Thus, for given $K$, the average number of dropped packets is given by $U(K)=K\left(1-\alpha_{L}^{K-1}\right)$. From this, we have

$$
\lambda_{\mathrm{d}}=\mathbb{E}[U(K)]=\lambda\left(1-e^{-\frac{\lambda}{L}}\right)=\lambda-\bar{\lambda} .
$$

Clearly, from (23) and (26), it can be shown that

$$
\lambda_{\mathrm{n}}=\bar{\lambda}=\lambda e^{-\frac{\lambda}{L}} \leq L e^{-1}
$$

and the maximum throughput of the IRT system can be

$$
\eta_{\text {irt }}=\max _{\lambda} \bar{\lambda}=L e^{-1}
$$

where the upper-bound is achieved when $\lambda=L$. That is, in the IRT system, with a finite $M$, it seems that a maximum throughput of $L e^{-1}$ is achievable. However, if there are a large number of PC-free devices, the SINR becomes low and the number of PiSs increases, which may make the IRT system unstable. Thus, for a stable IRT system, it is expected that any PC-free devices receive ACK of Type II after a finite number of re-transmissions. Thus, we now focus on the derivation of key conditions for a finite number of PiSs and the throughput of stable IRT.

Let $A(t)$ denote the number of new data packets arrived at slot $t$. That is, $A(t)$ is the number of new PC-free devices in 
slot $t$. Then, it can be shown that $\mathbb{E}[A(t)]=\mathbb{E}\left[K \alpha_{L}^{K-1}\right]=\bar{\lambda}$, i.e.,

$$
A(t) \sim \operatorname{Pois}(\bar{\lambda})
$$

We assume that $A(t)$ is an independent Poisson random variable with mean $\lambda-\bar{\lambda}$. In addition, denote by $\bar{K}(t)$ the total number of the data packets in slot $t$. In the IRT system, denote by $W_{q, t^{\prime}}$ the number of the $q$ th PiS that is first transmitted at time slot $t^{\prime}$. Thus, the number of the $q$ th PiS at time slot $t$ is $W_{q, t-q}$, and it can be shown that

$$
\bar{K}(t)=A(t)+\sum_{q=1}^{\infty} W_{q, t-q} .
$$

Note that in the conventional system, $\bar{K}(t)=A(t)$ as no immediate re-transmissions are considered.

Since all the PiSs are copies of the 0th PiS, the BS can exploit the RTD gain by combining all the PiSs and the resulting SINR is a sum of the SINRs of individual PiSs [26]. Thus, in slot $t$, the SINR of a data packet from a PC-free device with $(q+1)$-time transmissions is given by

$$
\begin{aligned}
\operatorname{SINR}_{\text {rtd }} & =\frac{M}{\bar{K}(t) b_{1}+b_{0}}+\ldots+\frac{M}{\bar{K}(t-q) b_{1}+b_{0}} \\
& =\frac{M}{\bar{K}(t) b_{1}+b_{0}}\left(1+\sum_{i=1}^{q} \frac{\bar{K}(t) b_{1}+b_{0}}{\bar{K}(t-i) b_{1}+b_{0}}\right) \\
& \approx \operatorname{SINR}(\bar{K}(t), q),
\end{aligned}
$$

where

$$
\operatorname{SinR}(K, q)=\frac{(q+1) M}{K b_{1}+b_{0}} .
$$

Here, the approximation in (31) is valid if $\bar{K}(t)$ is not significantly varying over $t$, i.e., $\bar{K}(t) \approx \cdots \approx \bar{K}(t-q)$. With RTD, it is clear that the SINR of PiS increases with $q$ provided that $K$ is fixed or bounded. Thus, for a sufficiently large $q$, the packet can be decoded and $W_{q, t-q}$ becomes 0 . In other words, the total number of packets in slot $t, \bar{K}(t)$ in (30), can be bounded.

Lemma 1: With RTD, suppose that the SINR of a packet with $(q+1)$ th transmissions is given by (32). Then, the number of PiSs in slot $t$ becomes

$$
\bar{K}(t)=A(t)+\sum_{q=1}^{Q_{\Gamma}(\bar{K}(t-1))} A(t-q),
$$

where

$$
Q_{\Gamma}(K)=\min _{q}\{q: \operatorname{SINR}(K, q) \geq \Gamma\} .
$$

Proof: $\operatorname{Since} \operatorname{SINR}(K, q)>\operatorname{SINR}(K, q-1)$, we can show that

$$
\operatorname{SINR}\left(K, Q_{\Gamma}(K)\right) \geq \Gamma \text { and } \operatorname{SINR}\left(K, Q_{\Gamma}(K)-1\right)<\Gamma .
$$

Thus, according to (35), in slot $t-1$, the $Q_{\Gamma}(\bar{K}(t-1))$ th PiS is decodable, but the $\left(Q_{\Gamma}(\bar{K}(t-1))-q\right)$ th PiS is not, where $q=1, \ldots, Q_{\Gamma}(\bar{K}(t-1))$. As a result, in slot $t$, we can show that

$$
\sum_{q=1}^{\infty} W_{q, t-q}=\sum_{q=1}^{\left(Q_{\Gamma}(\bar{K}(t-1))-1\right)+1} W_{q, t-q}
$$

or

$$
\bar{K}(t)=A(t)+\sum_{q=1}^{Q_{\Gamma}(\bar{K}(t-1))} W_{q, t-q} .
$$

Since the number of the 0th PiSs in slot $t$ is $A(t)$, i.e., $W_{0, t}=A(t), W_{q, t-q}$ is either $A(t-q)$ or 0 . That is, $W_{q, t-q}$ can be replaced with $A(t-q)$ unless the $q$ th PiSs are decodable. Thus, letting $W_{q, t-q}=A(t-q)$ in (36), we can obtain (33), which completes the proof.

From (33), it can be shown that if $\bar{K}(t)$ increases with $t$, it results in the decrease of SINR and the increase of $Q_{\Gamma}(\bar{K}(t))$ based on the definition of $Q_{\Gamma}(K)$ in (34). This implies that $\bar{K}(t)$ can be unbounded and the IRT system becomes unstable. To avoid it, it is necessary to find stability conditions. To this end, we consider Foster-Lyapunov criteria [20] [21].

In (33), since $\bar{K}(t)$ depends on $\bar{K}(t-1)$, it can be seen as a Markov chain. Since $A(t) \in\{0,1, \ldots\}$, the state space of $\bar{K}(t)$ becomes

$$
\bar{K}(t) \in \mathbb{Z}^{*} \triangleq\{0,1, \ldots\},
$$

where $\mathbb{Z}^{*}$ is the set of non-negative integers.

Lemma 2: If

$$
M>\bar{\lambda} \Gamma b_{1}=\lambda e^{-\frac{\lambda}{L}} \Gamma\left(1+\frac{1}{\gamma}\right),
$$

$\bar{K}(t)$ in (33) is a positive recurrent Markov chain.

Proof: Since $\bar{K}(t) \geq 0$, we assume that $\bar{K}(t)$ itself is a Lyapunov function. From (34),

$$
Q_{\Gamma}(K)=\left\lceil\frac{\Gamma\left(K b_{1}+b_{0}\right)}{M}-1\right\rceil .
$$

From (29), (33), and (38), we have

$$
\begin{aligned}
\mathbb{E}[\bar{K}(t) \mid \bar{K}(t-1)=k] & =\bar{\lambda}\left(1+Q_{\Gamma}(k)\right) \\
& =\bar{\lambda}\left(\frac{\Gamma\left(k b_{1}+b_{0}\right)}{M}+\delta\right) \\
& =\frac{\bar{\lambda} \Gamma b_{1}}{M} k+\frac{\bar{\lambda} \Gamma b_{0}}{M}+\delta,
\end{aligned}
$$

where $\delta \in[0,1)$, since $\lceil x\rceil$ can be expressed as $x+\delta$.

Suppose that (37) holds. Then, since $\frac{\bar{\lambda} \Gamma b_{1}}{M}>1$, there exists a finite $k^{*}$ as follows:

$$
k^{*}=\min _{k}\left\{k: \frac{\bar{\lambda} \Gamma b_{1}}{M} k+\frac{\bar{\lambda} \Gamma b_{0}}{M}>k\right\} \text {. }
$$

Let

$$
\mathcal{K}=\left\{0,1, \ldots, k^{*}\right\},
$$

which is a finite set. Then, for $\epsilon>0$, it can be shown that

$$
\begin{aligned}
& d(k)=\mathbb{E}[\bar{K}(t) \mid \bar{K}(t-1)=k]-k<-\epsilon, k \in \mathcal{K}^{c} \\
& d(k)<\frac{\bar{\lambda} \Gamma b_{0}}{M}+1-\epsilon, k \in \mathcal{K},
\end{aligned}
$$

where $\mathcal{K}^{c}=\mathbb{Z}^{*} \backslash \mathcal{K}$. According to [20, Proposition D.1], (42) implies that $\bar{K}(t)$ is a positive recurrent Markov chain.

Consequently, with stable IRT, from (37), the maximum throughput in (28) is to be replaced by

$$
\eta_{\text {irt }}=\min \left\{L e^{-1}, \frac{M}{\Gamma\left(1+\frac{1}{\gamma}\right)}\right\} .
$$


The arrival rate corresponding to $\eta_{\text {irt }}$ is denoted by $\lambda=\lambda_{\text {irt }}$. Note that the throughput of IRT is $\bar{\lambda}$ as mentioned earlier as long as (37) holds.

In Fig. 3, we show $\lambda_{\mathrm{n}}$ as a function of $\lambda$ for the conventional and IRT systems when $M=100, L=64$, and $\gamma=\Gamma=6$ $\mathrm{dB}$. In this case, $\left\lfloor K_{\Gamma}\right\rfloor$ is 20 . In addition, it can be found that $\eta_{\text {con }}=13.13$ and $\eta_{\text {irt }}=20.07$, i.e., the IRT system has a higher throughput than the conventional system. Note that in the IRT system, if $\lambda>\lambda_{\text {irt }}$, it becomes unstable (i.e., $\bar{K}(t) \rightarrow$ $\infty)$.

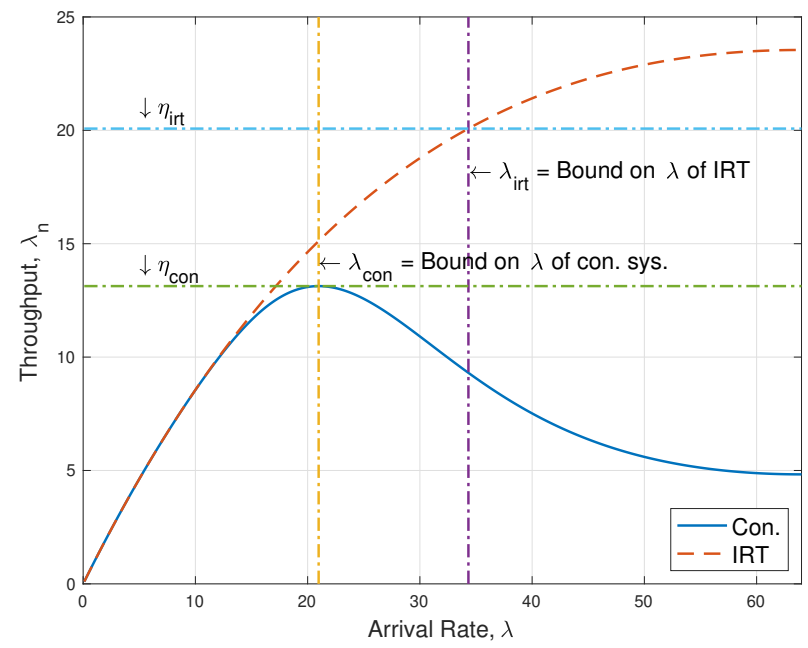

Fig. 3. Throughput, $\lambda_{n}$, as a function of $\lambda$ for the conventional and IRT systems when $M=100, L=64$, and $\gamma=\Gamma=6 \mathrm{~dB}$.

Lemma 3: If (37) holds,

$$
\eta_{\text {irt }} \geq \eta_{\text {con }}
$$

Proof: From (25), we have

$$
\eta_{\text {con }} \leq \bar{\lambda} \text {. }
$$

From (43), $\eta_{\text {irt }}$ is either $\frac{M}{\Gamma b_{1}}$ or $L e^{-1}$. If $\eta_{\text {irt }}=L e^{-1}$, since $\bar{\lambda}=L e^{-1}$, from (45), we have (44). On the other hand, suppose that $\eta_{\mathrm{irt}} \rightarrow \frac{M}{\Gamma b_{1}}$. If (37) holds, we have $\frac{M}{\Gamma b_{1}}>\bar{\lambda}$, which implies (44). This completes the proof.

In Fig. 4, the maximum throughputs of the conventional and the IRT systems are shown as functions of $M$ and $\Gamma$ when $L=64$ and $\gamma=6 \mathrm{~dB}$. Unless $M$ is sufficiently large (for a fixed $\Gamma$ ) or $\Gamma$ is sufficiently low (for a fixed $M$ ), it is shown that $\eta_{\text {irt }}$ is higher than $\eta_{\text {con }}$. However, for a large $M / \Gamma$, both the systems have the same maximum throughput, $L e^{-1}$, which is limited by the number of preambles.

Note that since $\bar{\lambda} \leq L e^{-1}$, a sufficient condition for (37) can be found as

$$
M>L e^{-1} \Gamma b_{1},
$$

which is independent of $\lambda$. In addition, since $\bar{\lambda} \leq \lambda$, another sufficient condition is given by

$$
M>\lambda \Gamma b_{1},
$$

which is independent of $L$.

Since $\bar{K}(t)$ is a positive recurrent Markov chain when (37) holds, $\bar{K}(t)$ has a unique stationary distribution [27] and $\mu_{\bar{K}}=$

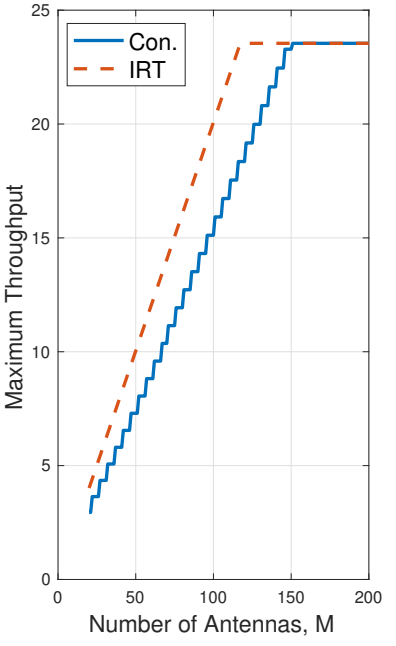

(a) (b)

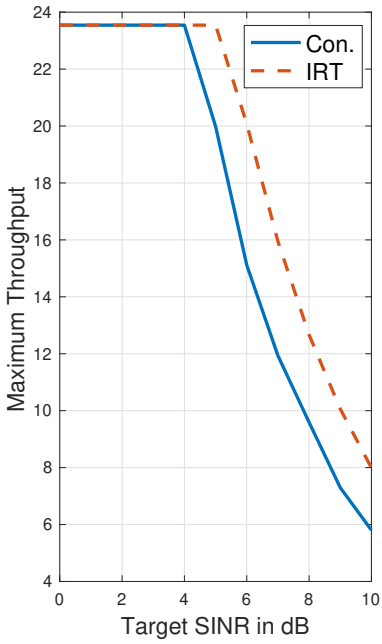

Fig. 4. The maximum throughputs of conventional and IRT systems: (a) throughput versus $M$ with $L=64$ and $(\Gamma, \gamma)=(6,6)$ in $\mathrm{dB}$; (b) throughput versus $\Gamma$ with $M=100, L=64$ and $\gamma=6 \mathrm{~dB}$.

$\mathbb{E}[\bar{K}(t)]$ exists. Let $\pi(k)=\operatorname{Pr}(\bar{K}(t)=k)$ be the stationary distribution. From (33), it can be shown that

$$
\mu_{\bar{K}}=\sum_{k} k \pi(k)=\bar{\lambda}\left(1+\sum_{k} Q_{\Gamma}(k) \pi(k)\right) .
$$

Unfortunately, it is difficult to find $\mu_{\bar{K}}$ as $Q_{\Gamma}(k)$ is a nonlinear function of $k$. However, with an approximation of the ceiling function, i.e., $\lceil x\rceil \approx x+\frac{1}{2}, Q_{\Gamma}(k)$ in (38) can be approximated as

$$
Q_{\Gamma}(k) \approx \frac{\Gamma\left(k b_{1}+b_{0}\right)}{M}-1+\frac{1}{2},
$$

which can be used in (48) to find the following approximation of $\mu_{\bar{K}}$ :

$$
\mu_{\bar{K}} \approx \frac{\bar{\lambda}\left(\frac{M}{2}+\Gamma b_{0}\right)}{M-\bar{\lambda} \Gamma b_{1}}
$$

We can also have bounds on $\mu_{\mathrm{K}}$ as follows.

Lemma 4: With $\bar{K}(t)$ is in (33), we have

$$
\bar{\lambda} \leq \mu_{\bar{K}} \leq \frac{\bar{\lambda}\left(M+\Gamma b_{0}\right)}{M-\bar{\lambda} \Gamma b_{1}} .
$$

Proof: From (39), since $Q_{\Gamma}(K) \geq 0$, we can have the lower bound in (50) with $Q_{\Gamma}(K)=0$. For the upper-bound, it can be shown that

$$
\mathbb{E}[\bar{K}(t) \mid \bar{K}(t-1)] \leq \frac{\bar{\lambda} \Gamma b_{1}}{M} \bar{K}(t-1)+\frac{\bar{\lambda} \Gamma b_{0}}{M}+1 .
$$

Then, we have

$$
\begin{aligned}
\mu_{\bar{K}} & =\mathbb{E}[\mathbb{E}[\bar{K}(t) \mid \bar{K}(t-1)]] \\
& \leq \frac{\bar{\lambda} \Gamma b_{1}}{M} \mu_{\bar{K}}+\frac{\bar{\lambda} \Gamma b_{0}}{M}+1,
\end{aligned}
$$

which results in the upper bound in (50).

According to Little's law [20], the average number of retransmissions till ACK of Type II for a PC-free device is bounded as follows:

$$
1 \leq \tau_{\mathrm{PiS}}=\frac{\mu_{\bar{K}}}{\bar{\lambda}} \leq \frac{M+\Gamma b_{0}}{M-\bar{\lambda} \Gamma b_{1}} .
$$


Using the approximation in (49), the approximate average number of re-transmissions can also be obtained as follows:

$$
\tilde{\tau}_{\mathrm{PiS}}=\max \left\{1, \frac{\frac{M}{2}+\Gamma b_{0}}{M-\bar{\lambda} \Gamma b_{1}}\right\} .
$$

For a short access delay, clearly, a small $\tau_{\mathrm{PiS}}$ is desirable.

\section{Simulation Results}

In Section V, we analyze the conventional and IRT systems with various assumptions and approximations. In particular, the approximations of SINR play a key role in simplifying the analysis (e.g., (21)) and finding the condition for stable IRT in (50) (e.g., (31)). In this section, we present simulation results and show that the analysis results agree with them. In simulations, new arrivals are generated according to (18) and the v's are generated as independent CSCG random vectors according to the assumption of $\mathbf{A}$ ). The actual SINR in (9) is used for successful decoding in both the systems and immediate re-transmissions in the IRT system.

Fig. 5 (a) shows the throughputs of the conventional and IRT systems for different values of arrival rate, $\lambda$, when $M=100$, $L=64$, and $\gamma=\Gamma=6 \mathrm{~dB}$. Thanks to RTD in the IRT system, we can see that the throughput of the IRT system is higher than that of the conventional system. In Fig. 5 (b), it is shown that $\tau_{\text {PiS }}$ of the IRT system increases with $\lambda$, and as $\lambda$ approaches $\lambda_{\text {irt }}, \tau_{\text {PiS }} \rightarrow \infty$ (i.e., the IRT system becomes unstable). Consequently, $\lambda$ has to be lower than $\lambda_{\text {irt }}$. Note that the upper-bound on the and approximation of $\tau_{\mathrm{PiS}}$ shown in Fig. 5 (b) are obtained from (53) and (54), respectively.

In Fig. 6 (a), the throughputs of the conventional and IRT systems for different values of threshold SINR, $\Gamma$, are shown when $M=100, L=64, \lambda=20$, and $\gamma=6 \mathrm{~dB}$. The bound on $\Gamma$ is $\frac{M}{\lambda b_{1}}=7.37$ (dB) from (37). In the IRT system, with $\Gamma<\frac{M}{\lambda b_{1}}$, its throughput is $\bar{\lambda}=\lambda e^{-\lambda / L}=14.63$. However, the throughput of the conventional system becomes lower than 14.63 when $\Gamma>4$ dB. Fig. 6 (b) shows $\tau_{\text {PiS }}$ as a function of $\Gamma$. As $\Gamma$ approaches $\frac{M}{\lambda b_{1}}$, we can see that $\tau_{\mathrm{PiS}}$ approaches $\infty$. Thus, for stable IRT, $\Gamma$, has to be lower than $\frac{M}{\lambda b_{1}}$.

To see the impact of the number of antennas on the performance, simulations are carried out with different values of $M$ and the results are shown in Fig. 7 when $\lambda=20$, $L=64$, and $\gamma=\Gamma=6 \mathrm{~dB}$. The bound on $M$ is $\bar{\lambda} \Gamma b_{1}=72.88$ from (37). As shown in Fig. 7 (a), if $M$ is not too large (e.g., $M<140$ ), the throughput of the conventional system is lower than $\bar{\lambda}=14.63$, which is the throughput of stable IRT. In Fig. 7 (b), $\tau_{\mathrm{PiS}}$ of IRT is shown, where we can see that $\tau_{\mathrm{PiS}}$ becomes finite if $M>\bar{\lambda} \Gamma b_{1}=72.88$.

In grant-free random access with massive MIMO, the number of preambles, $L$, is one of key parameters. Since the probability of PC decreases with $L$, a large $L$ is expected for a higher throughput. In Fig. 8, the impact of $L$ on the performance is shown with $M=100, \lambda=20$, and $\gamma=\Gamma=6$ $\mathrm{dB}$. In this case, since

$$
\bar{\lambda} \Gamma b_{1} \leq \lambda \Gamma b_{1}=99.62<M=100,
$$

i.e., (47) holds, the IRT system becomes stable for any value of $L$. Thus, the throughput of IRT, $\bar{\lambda}=\lambda e^{-\frac{\lambda}{L}}$, increases

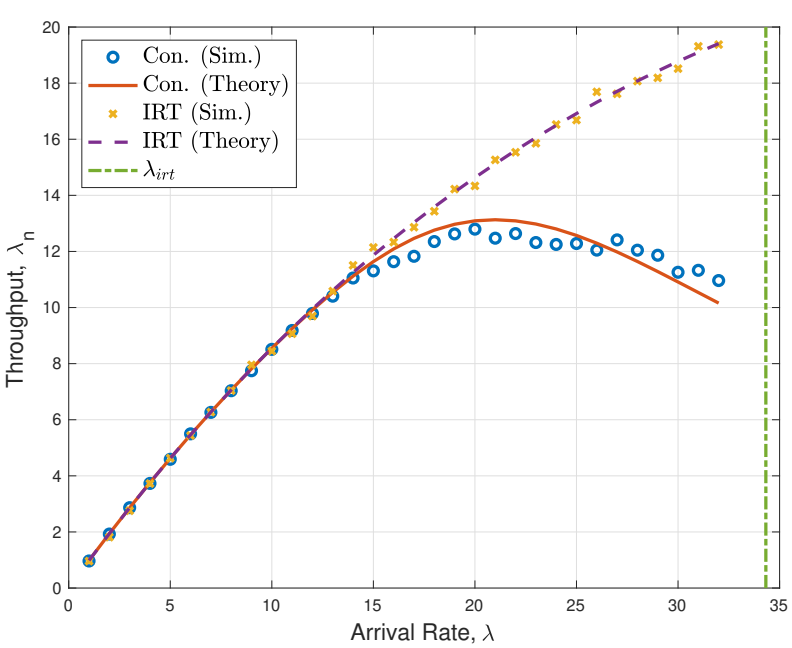

(a)

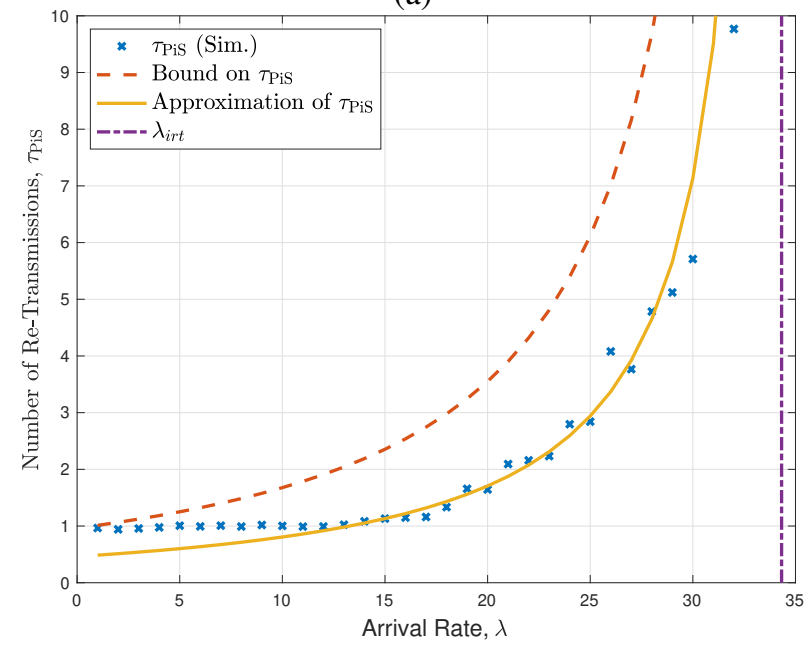

(b)

Fig. 5. Performance of the conventional and IRT systems for different values of arrival rate, $\lambda$, when $M=100, L=64$, and $\gamma=\Gamma=6 \mathrm{~dB}$ : (a) throughput versus $\lambda$; (b) $\tau_{\mathrm{PiS}}$ versus $\lambda$.

with $L$ as shown in Fig. 8 (a). On the other hand, in the conventional system, the increase of $L$ results in the increase of PC-free devices that leads to the decrease of SINR and increase of the probability of unsuccessful decoding. As a result, the throughput does not increase with $L$ once $L$ is sufficiently large as shown in Fig. 8 (a). In the IRT system, as mentioned earlier, since the increase of $L$ results in the increase of $\bar{K}(t), \tau_{\mathrm{PiS}}$ can increase with $L$ as shown in Fig. 8 (b). Thus, there is a trade-off between $\tau_{\text {PiS }}$ (or delay) and throughput in terms of $L$ in the IRT system.

\section{CONCLUding ReMARKS}

In this paper, in order to improve the throughput of grantfree random access with massive MIMO, we proposed an approach that is based on immediate re-transmissions for RTD gain. In particular, for a PC-free active device, since its channel vector is already estimated, no preamble transmissions are required in immediate re-transmissions. As a result, without increasing the probability of PC in subsequent slots, the 


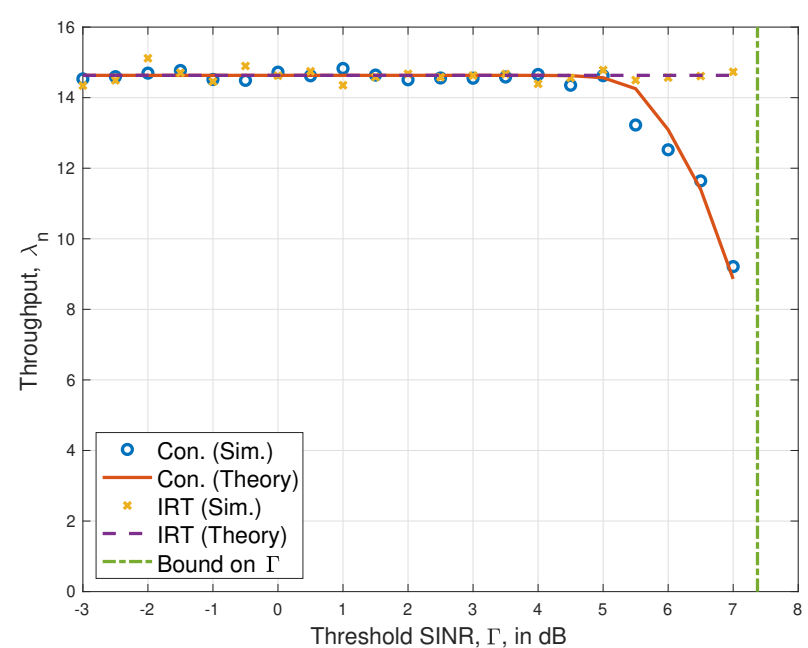

(a)

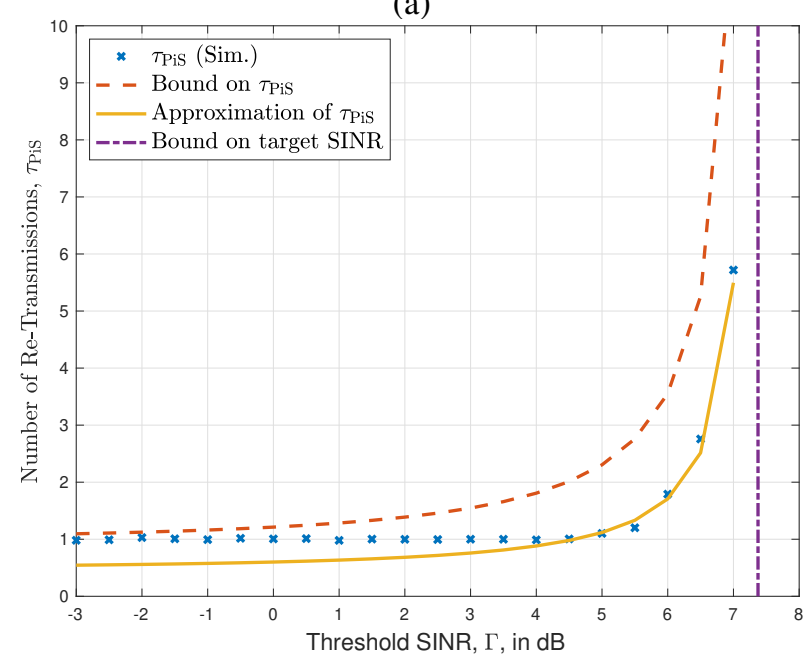

(b)

Fig. 6. Performance of the conventional and IRT systems for different values of threshold SINR, $\Gamma$, when $M=100, L=64$, and $\gamma=\Gamma=6 \mathrm{~dB}$ : (a) throughput versus $\Gamma$; (b) $\tau_{\mathrm{PiS}}$ versus $\Gamma$.

SINR could be increased by immediate re-transmissions for successful decoding that leads to improved throughput.

The proposed system with a finite number of antennas has been analyzed using certain approximations of SINR with RTD and the resulting analysis provided key conditions in terms of the number of antennas, $M$, and the threshold SINR, $\Gamma$, to avoid unstable systems where the number of retransmissions can be unbounded. In addition, the throughput of the proposed system was obtained, which is shown to be higher than that of the conventional system. Since the analysis was based on approximations of SINR, simulations have been carried out and it was shown that analysis results reasonably match simulation results.

In this paper, we mainly focused on the throughput of the proposed system without considering any re-transmission strategies for the packets of active devices with PC (i.e., backlogged packets). Optimal re-transmission strategies for backlogged packets with delay constraints and their delay analysis might be further research topics. In addition, the

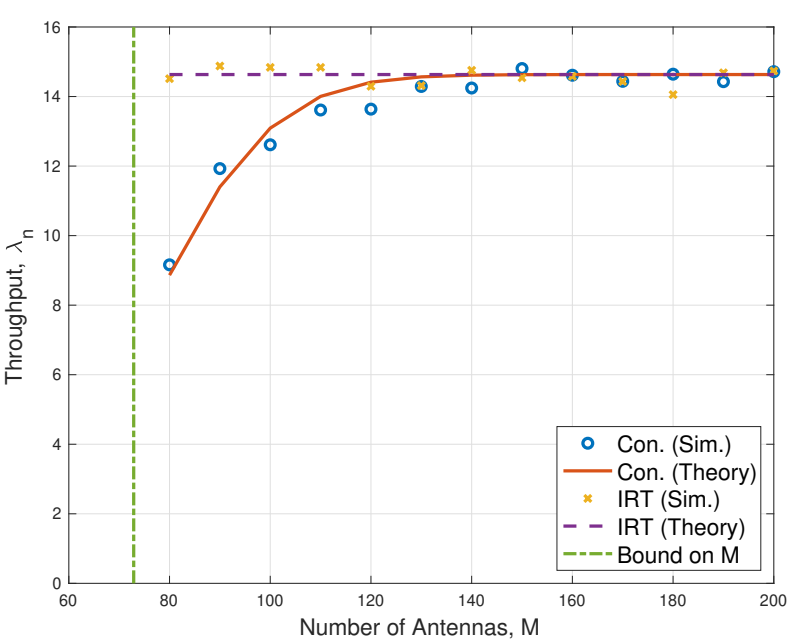

(a)

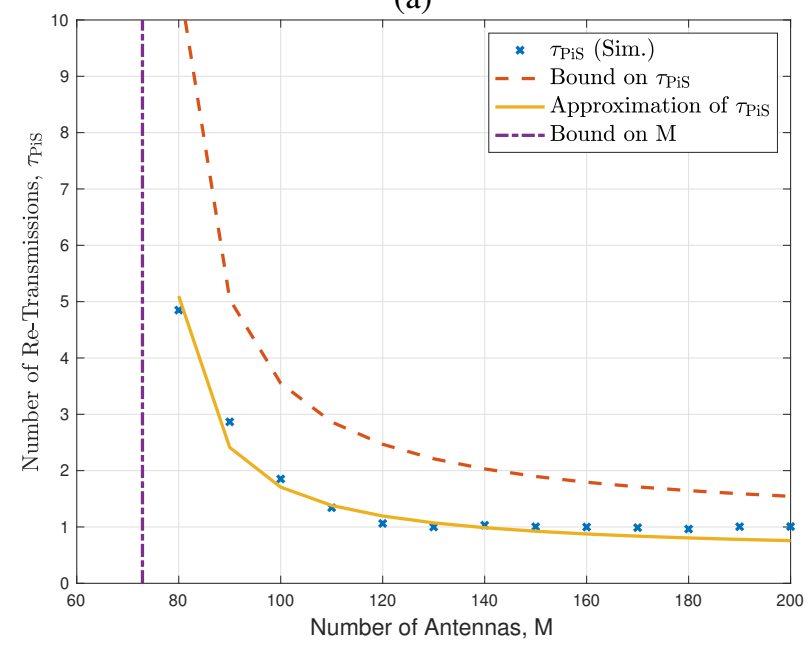

(b)

Fig. 7. Performance of the conventional and IRT systems for different values of the number of antennas, $M$, when $\lambda=20, L=64$, and $\gamma=\Gamma=6 \mathrm{~dB}$ : (a) throughput versus $M$; (b) $\tau_{\mathrm{PiS}}$ versus $M$.

proposed approach can be generalized with non-orthogonal preambles for a higher throughput, which is another further research topic.

\section{REFERENCES}

[1] J. Ding, M. Nemati, C. Ranaweera, and J. Choi, "IoT connectivity technologies and applications: A survey," IEEE Access, vol. 8, pp. 6764667673, 2020.

[2] C. Bockelmann, N. Pratas, H. Nikopour, K. Au, T. Svensson, C. Stefanovic, P. Popovski, and A. Dekorsy, "Massive machine-type communications in 5G: physical and MAC-layer solutions," IEEE Communications Magazine, vol. 54, pp. 59-65, Sep 2016.

[3] Z. Dawy, W. Saad, A. Ghosh, J. G. Andrews, and E. Yaacoub, "Toward massive machine type cellular communications," IEEE Wireless Communications, vol. 24, pp. 120-128, Feb 2017.

[4] N. Abramson, "THE ALOHA SYSTEM: Another alternative for computer communications," in Proceedings of the November 17-19, 1970, Fall Joint Computer Conference, AFIPS '70 (Fall), (New York, NY, USA), pp. 281-285, ACM, 1970.

[5] 3GPP TR 37.868 V11.0, Study on RAN improvments for machine-type communications, October 2011.

[6] 3GPP TS 36.321 V13.2.0, Evolved Universal Terrestrial Radio Access (E-UTRA); Medium Access Control (MAC) protocol specification, June 2016. 


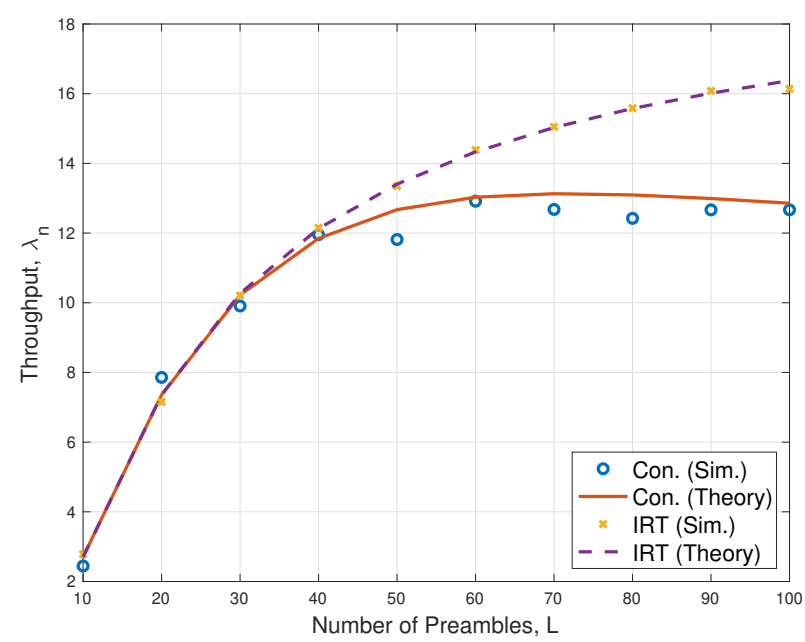

(a)

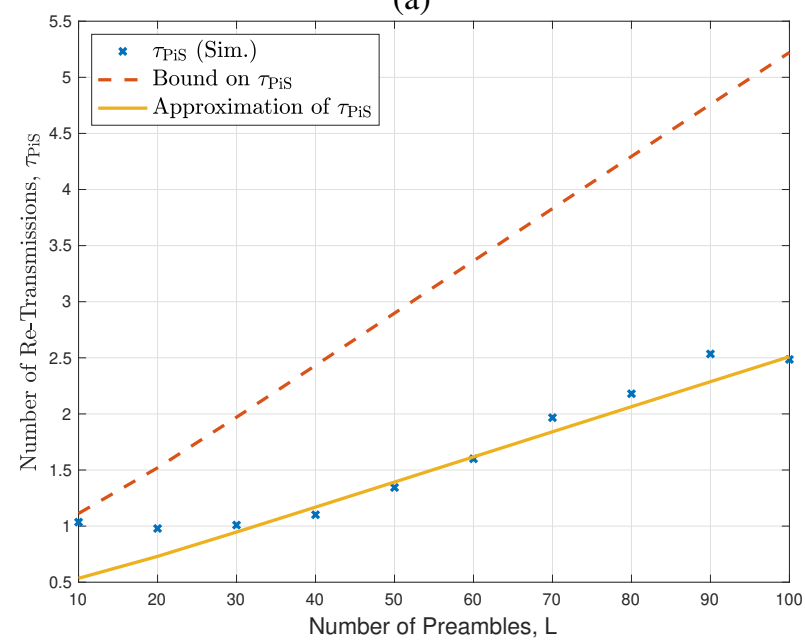

(b)

Fig. 8. Performance of the conventional and IRT systems for different values of the number of preambles, $L$, when $M=100, \lambda=20$, and $\gamma=\Gamma=6$ dB: (a) throughput versus $L$; (b) $\tau_{\mathrm{PiS}}$ versus $L$.

[7] C. H. Chang and R. Y. Chang, "Design and analysis of multichannel slotted ALOHA for machine-to-machine communication," in Proc. IEEE GLOBECOM, pp. 1-6, Dec 2015.

[8] J. Choi, "On the adaptive determination of the number of preambles in RACH for MTC," IEEE Communications Letters, vol. 20, pp. 13851388, July 2016.

[9] T. L. Marzetta, "Noncooperative cellular wireless with unlimited numbers of base station antennas," IEEE Trans. Wireless Communications, vol. 9, pp. 3590-3600, Nov. 2010.

[10] E. Bjrnson, J. Hoydis, and L. Sanguinetti, "Massive MIMO has unlimited capacity," IEEE Trans. Wireless Communications, vol. 17, pp. 574-590, Jan 2018.

[11] E. de Carvalho, E. Bjrnson, J. H. Srensen, E. G. Larsson, and P. Popovski, "Random pilot and data access in massive MIMO for machine-type communications," IEEE Trans. Wireless Communications, vol. 16, pp. 7703-7717, Dec 2017.

[12] K. Senel and E. G. Larsson, "Grant-free massive MTC-enabled massive MIMO: A compressive sensing approach," IEEE Trans. Communications, vol. 66, pp. 6164-6175, Dec 2018.

[13] L. Liu, E. G. Larsson, W. Yu, P. Popovski, C. Stefanovic, and E. de Carvalho, "Sparse signal processing for grant-free massive connectivity: A future paradigm for random access protocols in the Internet of Things," IEEE Signal Processing Magazine, vol. 35, pp. 88-99, Sept 2018.

[14] J. Ding, D. Qu, H. Jiang, and T. Jiang, "Success probability of grant-free random access with massive MIMO," IEEE Internet of Things J., vol. 6 , pp. 506-516, Feb 2019.
[15] J. Ding and J. Choi, "Performance analysis of massive MIMO assisted semi-grant-free random access," in IEEE CCNC, 2021, 2021 (Submitted).

[16] H. Jiang, D. Qu, J. Ding, and T. Jiang, "Multiple preambles for high success rate of grant-free random access with massive MIMO," IEEE Trans. Wireless Communications, vol. 18, pp. 4779-4789, Oct 2019.

[17] J. Ding, D. Qu, and J. Choi, "Analysis of non-orthogonal sequences for grant-free RA with massive MIMO," IEEE Trans. Communications, vol. 68, pp. 150-160, Jan 2020.

[18] J. Choi, "An approach to preamble collision reduction in grant-free random access with massive MIMO," IEEE Trans. Wireless Communications, 2020 (Submitted).

[19] Z. Chen and E. Bjrnson, "Channel hardening and favorable propagation in cell-free massive MIMO with stochastic geometry," IEEE Trans. Communications, vol. 66, pp. 5205-5219, Nov 2018.

[20] F. Kelly and E. Yudovina, Stochastic Networks. Cambridge University Press, 2014.

[21] B. Hajek, Random Processes for Engineers. Cambridge University Press, 2015.

[22] E. Bjrnson, E. G. Larsson, and M. Debbah, "Massive MIMO for maximal spectral efficiency: How many users and pilots should be allocated?," IEEE Trans. Wireless Communications, vol. 15, pp. 12931308, Feb 2016.

[23] E. Bjrnson, E. de Carvalho, J. H. Srensen, E. G. Larsson, and P. Popovski, "A random access protocol for pilot allocation in crowded massive MIMO systems," IEEE Trans. Wireless Communications, vol. 16, pp. 2220-2234, Apr 2017.

[24] L. Sanguinetti, A. A. DAmico, M. Morelli, and M. Debbah, "Random access in massive MIMO by exploiting timing offsets and excess antennas," IEEE Trans. Communications, vol. 66, pp. 6081-6095, Dec 2018.

[25] H. Q. Ngo, E. G. Larsson, and T. L. Marzetta, "Energy and spectral efficiency of very large multiuser MIMO systems," IEEE Trans. Communications, vol. 61, no. 4, pp. 1436-1449, 2013.

[26] G. Caire and D. Tuninetti, "The throughput of hybrid-ARQ protocols for the Gaussian collision channel," IEEE Trans. Inform. Theory, vol. 47, pp. 1971-1988, July 2001.

[27] J. Norris, Markov Chains. Cambridge Series in Statistical and Probabilistic Mathematics, Cambridge University Press, 1998. 